This item was submitted to Loughborough's Research Repository by the author.

Items in Figshare are protected by copyright, with all rights reserved, unless otherwise indicated.

\title{
Diffusive deposition of aerosols in a fibrous filter
}

PLEASE CITE THE PUBLISHED VERSION

https://doi.org/10.1016/j.jaerosci.2018.08.002

PUBLISHER

(c) Elsevier

VERSION

AM (Accepted Manuscript)

PUBLISHER STATEMENT

This paper was accepted for publication in the journal Journal of Aerosol Science and the definitive published version is available at https://doi.org/10.1016/j.jaerosci.2018.08.002.

\section{LICENCE}

CC BY-NC-ND 4.0

\section{REPOSITORY RECORD}

Clement, Charles F., and Sarah Dunnett. 2018. "Diffusive Deposition of Aerosols in a Fibrous Filter". figshare. https://hdl.handle.net/2134/34464. 


\title{
Diffusive Deposition of Aerosols in a Fibrous Filter
}

\author{
C. F. Clement ${ }^{\mathrm{a}}$ and S. J. Dunnett \\ a 15 Witan Way, Wantage, Oxon OX12 9EU, UK \\ ${ }^{\mathrm{b}}$ Department of Aeronautical and Automotive Engineering, Loughborough University, \\ Loughborough, Leics. LE11 3TU, UK
}

\begin{abstract}
Experimental deposition efficiencies for deposition from laminar flow in fibrous filters containing variable numbers of layers were obtained by Jackiewicz et al (2008). In the work presented here a particular case of their data for deposition onto filters with 1 to 9 layers is analysed in detail. Its dependence on layer depth is shown to be remarkably similar to the dependence on length of diffusive deposition from similar laminar flow in a cylindrical tube of a chosen radius. The observed mean layer deposition is shown to be consistent with theoretical predictions of deposition by diffusion and interception in the standard cell model. A reason for a larger than average deposition on the first layer is that many fibres in that layer remove particles from the initial particle concentration.
\end{abstract}

Keywords: Filtration; fibrous; diffusional deposition

\section{Introduction}

A fibrous filter contains a tangled mass of fibres placed approximately perpendicular to the gas flow through them. Its efficiency, E, is the fraction of aerosol in the flow which is retained by deposition from the flow during its passage through the filter. Much work on understanding this deposition has been based on a cell model in which deposition on a single fibre is calculated (Kuwabara 1959, Dunnett and Clement 2006). Cells throughout the filter are assumed to be identical so that the initial efficiency of the filter with passage through $\mathrm{m}$ cells is just obtained from a product of cell efficiencies, $\eta$,

$\mathrm{E}=1-(1-\eta)^{\mathrm{m}}$

However, this approach will fail if, as a result of diffusion, the distribution of particles in the flow entering a cell changes significantly. The efficiency then becomes a function of distance travelled through the filter. Experiments to measure efficiency as a function of this distance were performed by constructing filters with different numbers of nominally identical layers of filter material by Jankiewicz et al (2008). They interpreted the depth dependent efficiency found in terms of "axial diffusion". In this paper, by comparing the efficiency variation with that occurring in laminar tube flow, we show that a much more convincing 
explanation is that particle diffusion in the flow is responsible. Where diffusional deposition dominates removal in a fibrous filter, its efficiency can be accurately modelled by deposition from laminar flow in a tube of a chosen radius.

\section{Method}

For both a filter and a tube we consider a laminar airflow with velocity U containing spherical particles of diameter $d_{p}$ or radius $R=d_{p} / 2$. Gas spaces between fibres in the filter and the tube diameter are much larger than $\mathrm{R}$ and the gas molecule mean free path (both $<1$ $\mu \mathrm{m})$, so that particles reach walls for deposition by diffusion which represents particle collisions with gas molecules. The particle diffusivity in air, D, is specified by

$\mathrm{D}=\frac{\mathrm{k}_{\mathrm{B}} \mathrm{TC}(\mathrm{Kn})}{6 \pi \mu \mathrm{R}}$

where $k_{B}$ is the Boltzmann constant, $T$ is the temperature, $\mu$ is the air viscosity, and the Cunningham correction factor, $\mathrm{C}(\mathrm{Kn})$, is specified in terms of the Knudsen number, $\mathrm{Kn}=$ gas-mean-free-path/ R, by

$$
\mathrm{C}(\mathrm{Kn})=1+\mathrm{Kn}\left[1.237+0.4 \mathrm{e}^{-1.1 / \mathrm{Kn}}\right]
$$

\section{Tube Flow}

A solution for diffusive particle deposition from laminar flow along a cylindrical tube of radius, a, was obtained by Gormley and Kennedy (1949) and is quoted by Williams and Loyalka (1991). The fractional deposition in a distance $\mathrm{x}$ along the tube is a function of the dimensionless parameter.

$$
\mathrm{z}=\frac{\mathrm{x}}{\mathrm{aPe_{T }}}
$$

where the Peclet number for the tube, $\mathrm{Pe}_{\mathrm{T}}$, is defined by,

$$
\mathrm{Pe}_{\mathrm{T}}=\frac{\mathrm{a} \mathrm{U}}{\mathrm{D}}
$$

Hence, in terms of the basic parameters,

$$
\mathrm{z}=\frac{\mathrm{xD}}{\mathrm{Ua}^{2}}
$$

The analytic solution for the deposition efficiency, E, takes the form of a series specified by eigenfunctions and Williams and Loyalka (1991) give the first 3 terms as 
$\mathrm{E}($ tube $)=1-0.8191 \mathrm{e}^{-7.312 \mathrm{z}}-0.0975 \mathrm{e}^{-44.62 \mathrm{z}}-0.0325 \mathrm{e}^{-113.8 \mathrm{z}}$

For small $\mathrm{z}$ (or $\mathrm{x}$ ) the series converges slowly, and additional terms are needed very close to $\mathrm{z}=0$ where $\mathrm{E}($ tube $)=0$. However, for $\mathrm{z}=0.03$, the lowest value needed here, any corrections would be less than $0.1 \%$ and they will be ignored here.

\section{Filter Flow and Deposition}

To determine the experimental conditions where particle diffusion limits deposition in a filter, we need to examine particle, flow and fibre parameters following previous work by Stechkina and Fuchs (1966) and Dunnett and Clement (2006) using a staggered parallel cylinder model for fibres in the filter. This involves the Peclet number associated with the fibre diameter, $\mathrm{d}$, which is

$$
\mathrm{Pe}=\frac{\mathrm{dU}}{\mathrm{D}}
$$

In terms of this number, a dimensionless diffusive boundary layer around the fibre is given by

$$
\delta=(4 \mathrm{k} / \mathrm{Pe})^{1 / 3}
$$

where we take the hydrodynamic factor, $\mathrm{k}$, appropriate to the model, to be given by

$$
k=-0.5 \ln (\alpha)-0.75+\alpha-\frac{\alpha^{2}}{4}
$$

where $\alpha$ is the packing fraction of fibres in the filter.

Following Stechkina and Fuchs (1966 ), the other dimensionless parameter which determines the type of solution for the particle transport equation including diffusion is the dimensionless ratio of the particle size to the fibre size,

$$
\kappa=\frac{2 \mathrm{R}}{\mathrm{d}}
$$

The criterion for diffusive deposition is that this ratio is less than the boundary layer ratio i.e. $\mathrm{S}=\frac{\kappa}{\delta}<1$

Possibly the best comparison we can make with the explicit data presented by Jankiewicz et al (2008) is with the measured efficiencies for filter no.2 shown in their figure 3, where initial filter efficiency was plotted as a function of particle diameter for different number of layers of the filter. Starting with the lowest efficiency for one layer shown in the figure, 0.376 , which corresponds to an estimated particle diameter of $d_{p}=0.233 \mu \mathrm{m}$. The other basic quantities for the experiments on this filter were $U=0.2 \mathrm{~ms}^{-1}, \mathrm{~d}=15 \mu \mathrm{m}, \alpha=$ 
0.156, and layer thickness, $\mathrm{x}_{\mathrm{L}}=1.28 \mathrm{~mm}$. Then, choosing NTP values for air at $20^{0} \mathrm{C}$ with $\mu=1.81510^{-5} \mathrm{~kg} \mathrm{~m}^{-1} \mathrm{~s}^{-1}$ and gas mean-free path $=0.06544 \mu \mathrm{m}$, we find $\mathrm{D}=1.76710^{-10} \mathrm{~m}^{2} \mathrm{~s}^{-1}$ to be the particle diffusivity, $\mathrm{Pe}=16,980, \kappa=0.0155$, and the parameter $\mathrm{s}=0.364$. Hence diffusion dominates interception in the deposition process, and we only need to choose a tube radius, a, to use in eq.(6), to compare the observed efficiencies with those for deposition from laminar tube flow given by eq.(7). As shown in Fig.1, where E(tube) is plotted as a function of the number of layers of filter alongside the experimental data, the choice of $\mathrm{a}=5.83 \mu \mathrm{m}$ results in a good fit to the observed efficiencies whose values, reproduced in Table 1, were obtained from measurements on figure 3 of Jankiewicz et al (2008).

$\begin{array}{cccccccccc}\text { Layers L } & 1 & 2 & 3 & 4 & 5 & 6 & 7 & 8 & 9 \\ \text { E(L,exp) } & 0.376 & 0.444 & 0.542 & 0.693 & 0.779 & 0.814 & 0.837 & 0.892 & 0.90\end{array}$

Table 1. Estimated filtration efficiencies from figure 3 of Jankiewicz et al (2008)

The radius of a Kuwabara cell in the experimental filter considered is $\frac{\mathrm{d}}{2 \alpha^{1 / 2}}=19 \mu \mathrm{m}$,

which is much larger than the fitted value of a. The explanation of why diffusive deposition is being so efficient is that the staggered random placements of the fibres in the flow continually breaks it up so that all parts of it are brought close to fibre surfaces. In one filter layer, the flow passes $\mathrm{x}_{\mathrm{L}} \alpha^{1 / 2} / \mathrm{d}_{\mathrm{f}} \sim 34$ cells for the filter we are considering.

Although the total efficiencies, E(L, exp), obtained by Jankiewicz et al (2008) given in Table 1 were obtained with different filters, we can get some idea of how the efficiency is changing with number of layers, $\mathrm{L}$, from adjacent values of $\mathrm{E}(\mathrm{L}$, exp) in the table. Denoting individual layer efficiencies by $E_{L}$ for $L=1$ to 9 , the total efficiency, $E(L, \exp )$ will arise from that measured for L-1 layers, E(L-1, exp), by adding layer L, when we have for $\mathrm{L}>1$,

$$
\mathrm{E}_{\mathrm{L}}=1-\frac{1-\mathrm{E}_{\mathrm{L}}(\mathrm{L}, \exp )}{1-\mathrm{E}_{\mathrm{L}}(\mathrm{L}-1, \exp )}
$$

Values calculated from the data in Table 1 are shown in Figure 2 and exhibit large fluctuations. However, we believe these fluctuations represent those in the total efficiencies, $\mathrm{E}\left(\mathrm{L}\right.$, exp), arising from the different filters. Actual mean efficiencies in $\mathrm{E}_{\mathrm{L}}$ for the same filter are probably closer to those for the tube, E(L, tube), calculated directly from eq.(7) which are also shown in Figure 2. Fibrous filter construction will always be statistically variable and fluctuations in the sum of layers will always be larger than that for individual layers. Direct 
measurements of $E_{L}$ by adding, or subtracting, single layers to, or from, the same filter would run into problems of allowing for efficiency changes with deposition.

It is important to realise that the behaviour shown of a larger values of $E_{L}$ and $E(L$ tube) for $L=1$ followed by a constant value for larger $L$ has a physical explanation in both cases. For the tube it is the initial uniform value of the particle concentration and the high removal rate by diffusion of particles close to the tube wall. In the case of the filter, it arises from the shadowing effect of fibres and the distance or time needed for the particle concentration to be approximately uniform across the filter. When the initial uniform particle concentration, $\mathrm{n}_{0}$, reaches the first row of fibres, it is only depleted very close to the fibre surfaces. Diffusion is very slow, and, by the time the flow reaches the second layer, we expect the depletion layer to have expanded by much less than $1 \mu \mathrm{m}$. Because of the staggering and randomness of the fibre placing, the flow adjacent to nearly all the next layer will again have particle concentration, $\mathrm{n}_{0}$. This effect of particle concentration adjacent to fibres being $\mathrm{n}_{0}$ will extend into many of the 34 cells in the initial experimental filter layer leading to a larger removal efficiency in this layer. Eventually, after passing through many cells, the combination of diffusion and the randomness of particle removal is expected to make the removal rate, or efficiency, approximately proportional to the mean particle concentration, $\mathrm{n}$, in the filter layer.

This argument for an enhanced shadowing effect in the initial filter layer can be made more quantitative by extending previous work (Stechkina and Fuchs (1966), Dunnett and Clement (2006)) based on the following diffusion equation for the particle concentration, $n$, in a cell as shown in Figure 3:

$$
\mathrm{U}_{\mathrm{r}} \frac{\partial \mathrm{n}}{\partial \mathrm{r}}+\frac{\mathrm{U}_{\theta}}{\mathrm{r}} \frac{\partial \mathrm{n}}{\partial \theta}=\mathrm{D}\left(\frac{\partial^{2} \mathrm{n}}{\partial \mathrm{r}^{2}}+\frac{1}{\mathrm{r}} \frac{\partial \mathrm{n}}{\partial \mathrm{r}}+\frac{1}{\mathrm{r}^{2}} \frac{\partial^{2} \mathrm{n}}{\partial \theta^{2}}\right)
$$

where $\mathrm{U}_{\mathrm{r}}$ and $\mathrm{U}_{\theta}$ are the components of air velocity in polar coordinates. Nondimensionalising distances with respect to fibre radius, velocities with respect to $U_{0}$, and $n$ with respect to $\mathrm{n}_{0}$, the particle concentration entering the cell. Equation (14) then becomes

$$
\mathrm{U}_{\mathrm{r}} \frac{\partial \mathrm{n}}{\partial \mathrm{r}}+\frac{\mathrm{U}_{\theta}}{\mathrm{r}} \frac{\partial \mathrm{n}}{\partial \theta}=\frac{2}{\operatorname{Pe}}\left(\frac{\partial^{2} \mathrm{n}}{\partial \mathrm{r}^{2}}+\frac{1}{\mathrm{r}} \frac{\partial \mathrm{n}}{\partial \mathrm{r}}+\frac{1}{\mathrm{r}^{2}} \frac{\partial^{2} \mathrm{n}}{\partial \theta^{2}}\right)
$$

For fibrous filtration the third term on the right hand side of equation (15) is much smaller than the other terms and can been neglected, and the equation reduces to

$$
\mathrm{U}_{\mathrm{r}} \frac{\partial \mathrm{n}}{\partial \mathrm{r}}+\frac{\mathrm{U}_{\theta}}{\mathrm{r}} \frac{\partial \mathrm{n}}{\partial \theta}=\frac{2}{\mathrm{Pe}}\left(\frac{\partial^{2} \mathrm{n}}{\partial \mathrm{r}^{2}}+\frac{1}{\mathrm{r}} \frac{\partial \mathrm{n}}{\partial \mathrm{r}}\right)
$$


subject to the boundary conditions that $n=1$ away from the fibre, except near $\theta=\pi$, and $n=0$ at the interception radius, which is $1+\kappa$ for a clean fibre, where $\kappa$ is given by eq.(11). For large Peclet numbers the particles diffuse to the fibre from a thin layer (boundary layer) adjacent to its surface. The thickness of this layer is much less than half the distance between the fibres, which gives the condition that $\delta<1 / \sqrt{\alpha}$ where $\delta$ is the non-dimensional thickness of the diffusion layer. In order to solve equation (16) it is necessary to apply a third boundary condition and previously (Dunnett and Clement 2006), we followed the work of Stechkina and Fuchs (1966) and considered the line $\theta=0$ where $U_{\theta}=0$. On this line equation (14) reduces to an ordinary differential equation,

$$
\frac{2}{\operatorname{Pe}} \frac{\mathrm{d}^{2} \mathrm{n}}{\mathrm{dr}^{2}}+\frac{\mathrm{dn}}{\mathrm{dr}}\left(\frac{2}{\mathrm{r} \cdot \mathrm{Pe}}-\mathrm{U}_{\mathrm{r}}\right)=0
$$

We then obtained analytic solutions along the line $\theta=0$ using an analytic form for Ur valid near the cylinder surface:

$$
\mathrm{U}_{\mathrm{r}}=\frac{\left(1-\frac{1}{\mathrm{r}^{2}}-2 \ln \mathrm{r}\right)}{2 \mathrm{k}}
$$

The boundary conditions for this solution are $n=1$ at $r=\infty$ and $n=0$ at $r=1+\kappa$.

It is clear that equation (17) also applies at $\theta=\pi$, but here, as the flow is away from the surface with $\mathrm{n}=0$, the solution is simply

$\mathrm{n}(\mathrm{r})=0$.

The particle concentration on the cell boundary, AD in figure 3, where the flow exits the cell $(\theta>\pi / 2)$ will be 1 except very close to $\theta=\pi$, and this concentration distribution will form the entrance distribution for the next cell. If, as expected, fibre positions are staggered with respect to the flow direction, concentrations being removed by subsequent fibres close to the filter entrance will remain at $n=1$, making the efficiency of the first layer substantially larger than that of later layers.

Finally, we compare the observed layer removal efficiencies with those predicted by cell theories based on staggered cells of Stechkina and Fuchs (1966) and Dunnett and Clement (2006). The single cell removal rates, $\eta$, from numerical theory (Dunnett and Clement 2006) agree with those given by the following analytic expressions (Stechkina and Fuchs 1966) for small $\alpha$. 
$\eta_{\mathrm{SF}}=\eta_{\mathrm{D}}+\eta_{\kappa}+\frac{1.24 \kappa^{2 / 3}}{(\mathrm{kPe})^{2 / 3}}$

where

$\eta_{\mathrm{D}}=2.9 \mathrm{k}^{-1 / 3} \mathrm{Pe}^{-2 / 3}+\frac{0.624}{\mathrm{Pe}}$

$\eta_{\kappa}=\frac{(1+\kappa) \ln (1+\kappa)}{k}-\frac{1+\kappa}{2 \mathrm{k}}+\frac{1}{2 \mathrm{k}(1+\kappa)}$

Calculations with above values of $\mathrm{k}$, Pe and $\kappa$ give

$\eta_{\mathrm{D}}=0.0063692, \eta_{\kappa}=0.0007528, \eta_{\mathrm{SF}}=0.0081548$.

For a filter layer of 34 cells, the expected efficiency would then be:

$\mathrm{E}_{\mathrm{S}-\mathrm{F}}=1-\left(1-\eta_{\mathrm{SF}}\right)^{34}=0.243$

This theoretical value resulting from diffusion and interception by fibres is shown in Fig. 2, and the agreement with the values obtained from our interpretation of the observations is certainly good enough to establish that traditional filtration is responsible for the observed results. The axial diffusion introduced by Jankiewicz et al (2008) to explain their results is certainly unnecessary in this case. In general, “axial diffusion” produced by backwards flow in materials with low porosity does not exist in fibrous filters whose porosity is between 0.8 and 1.

In conclusion, we have shown that particle removal by a fibrous filter with large values of Pe is remarkably similar to diffusive deposition in a tube of an appropriate radius. The experimental results of layer distribution reported by Jackiewicz et al (2008) can be explained by ordinary particle diffusion rather than axial diffusion which does not apply to highly porous filters. Layer filtration efficiencies fluctuate from the random nature of filter construction, but a higher filtration efficiency is expected in the first layer arising from a failure in the standard cell model. Close to the filter entrance, the particle flux entering a staggered cell will be effectively larger than the mean flux at the cell depth.

\section{References}

Dunnett, S.J. and Clement, C.F. (2006) A numerical study of the effects of loading from diffusive deposition on the efficiency of fibrous filters. J. Aerosol Sci. 37, 1116-1139. Gormley, P.G. and Kennedy, M. (1949) Diffusion from a stream flowing through a cylindrical tube. Proc. Roy. Irish Academy 52, 163.

Jackiewicz, A., Balazy, A. and Podgorski, A. (2008) Investigation of aerosol dispersion in fibrous filters. Polish Journal of Chemical Technology 10 pp66-72 
Kuwabara, S. (1959) The forces exerted by randomly distributed parallel circular cylinders or spheres in a viscous flow at small Reynolds numbers, J. Phys. Soc. Japan, 14, pp527-532. Stechkina, I.B. and Fuchs, N.A. (1966) Studies on fibrous aerosol filters -I. Calculations of diffusional deposition of aerosols in fibrous filters, Ann. Occup. Hyg., 9, pp59-64.

Williams, M.M.R. and Loyalka, S.K. (1991) Aerosol Science Theory and Practice, Pergamon (Oxford) p.333.

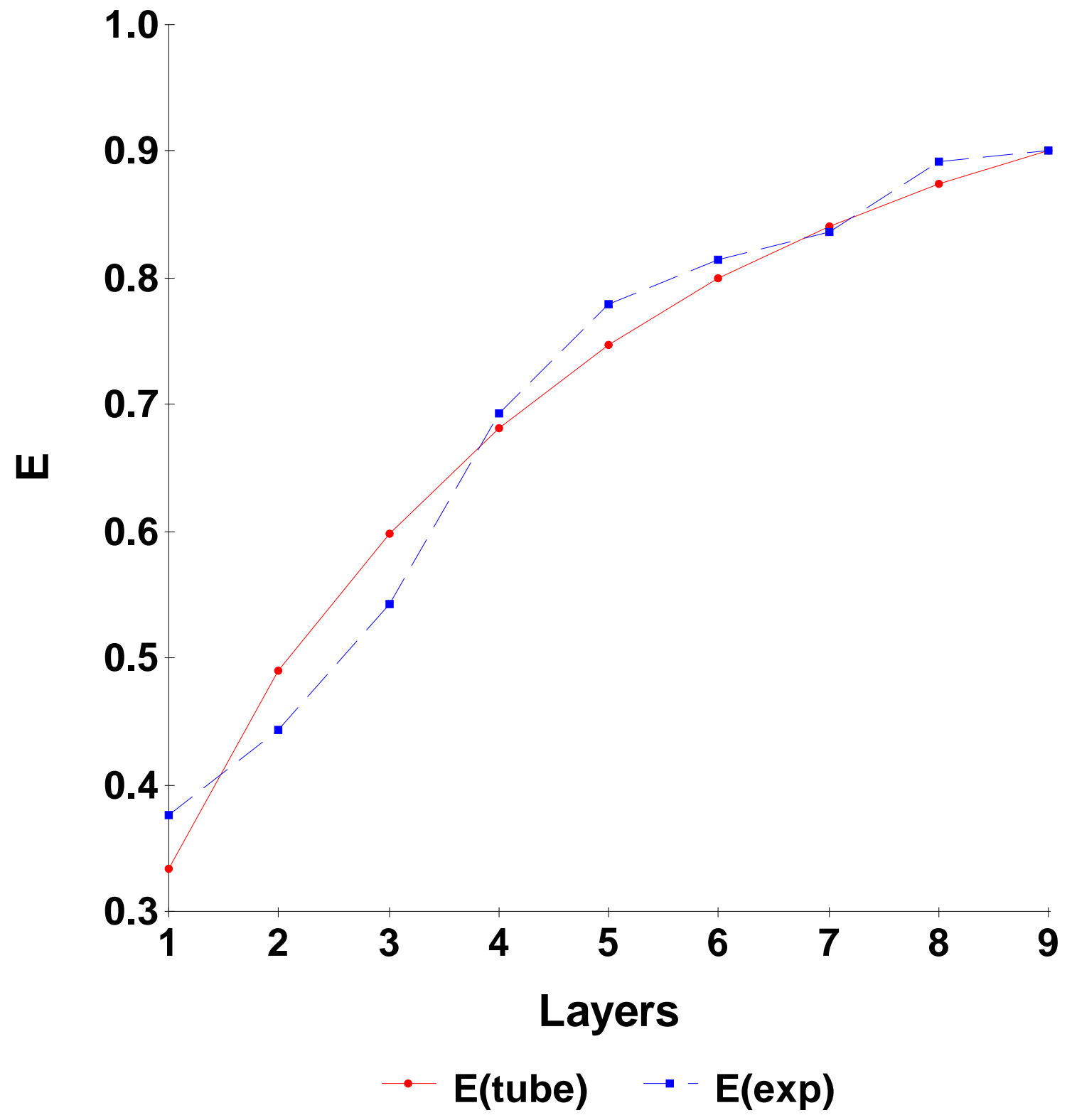

Fig.1. Particle deposition efficiencies, E(exp), observed for a fibrous filter with a thickness of the number of layers shown, compared to theoretical predictions, E(tube), of total diffusive 
deposition on the walls of a tube of the same length as the filter thickness and with the same laminar air flow. The tube radius was chosen to fit the data.

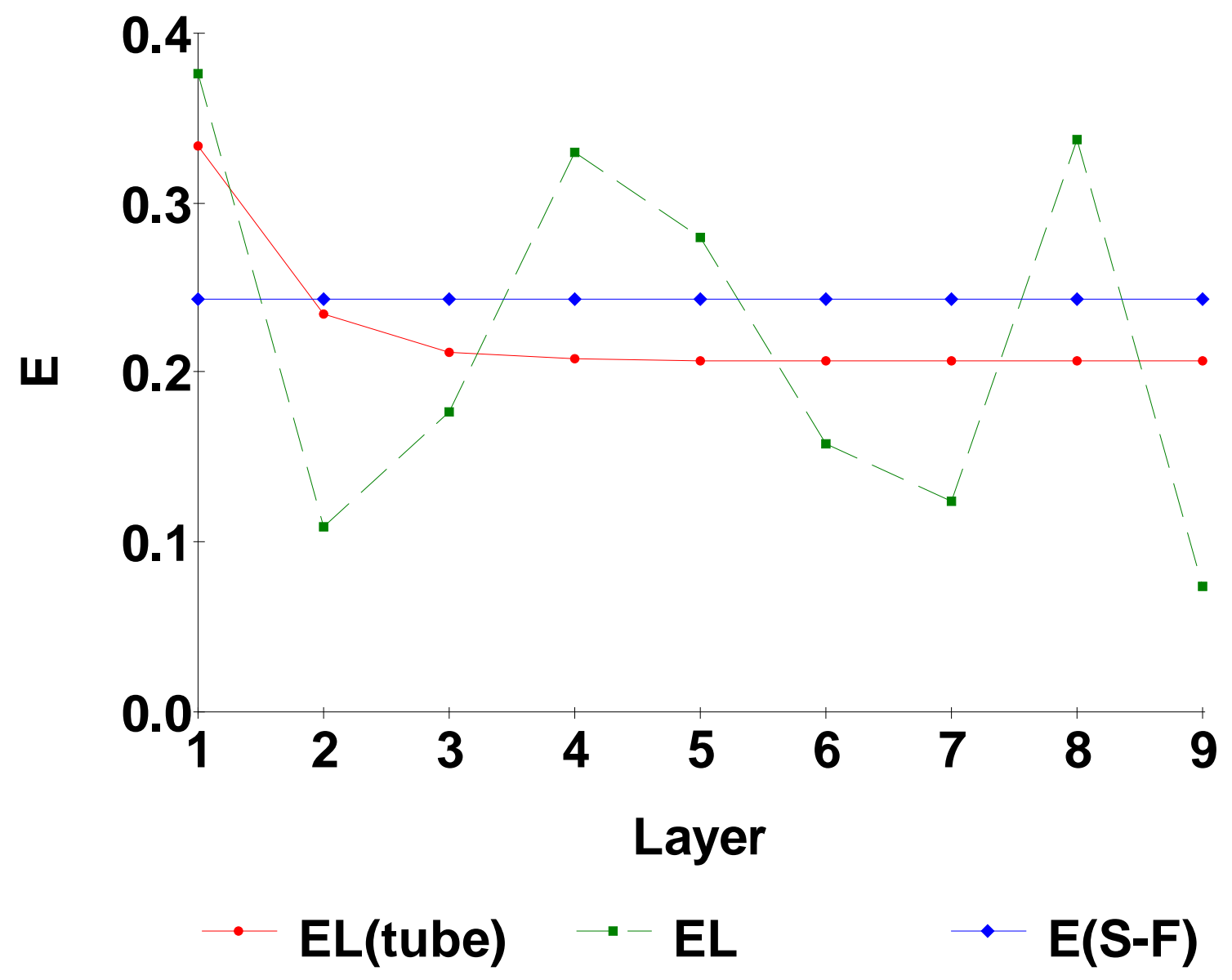

Fig. 2. Deposition efficiencies of individual layers for the tube, the filter (measured from different filters), and the value, E(S-F), from the theory of Stechkina and Fuchs (1966). 

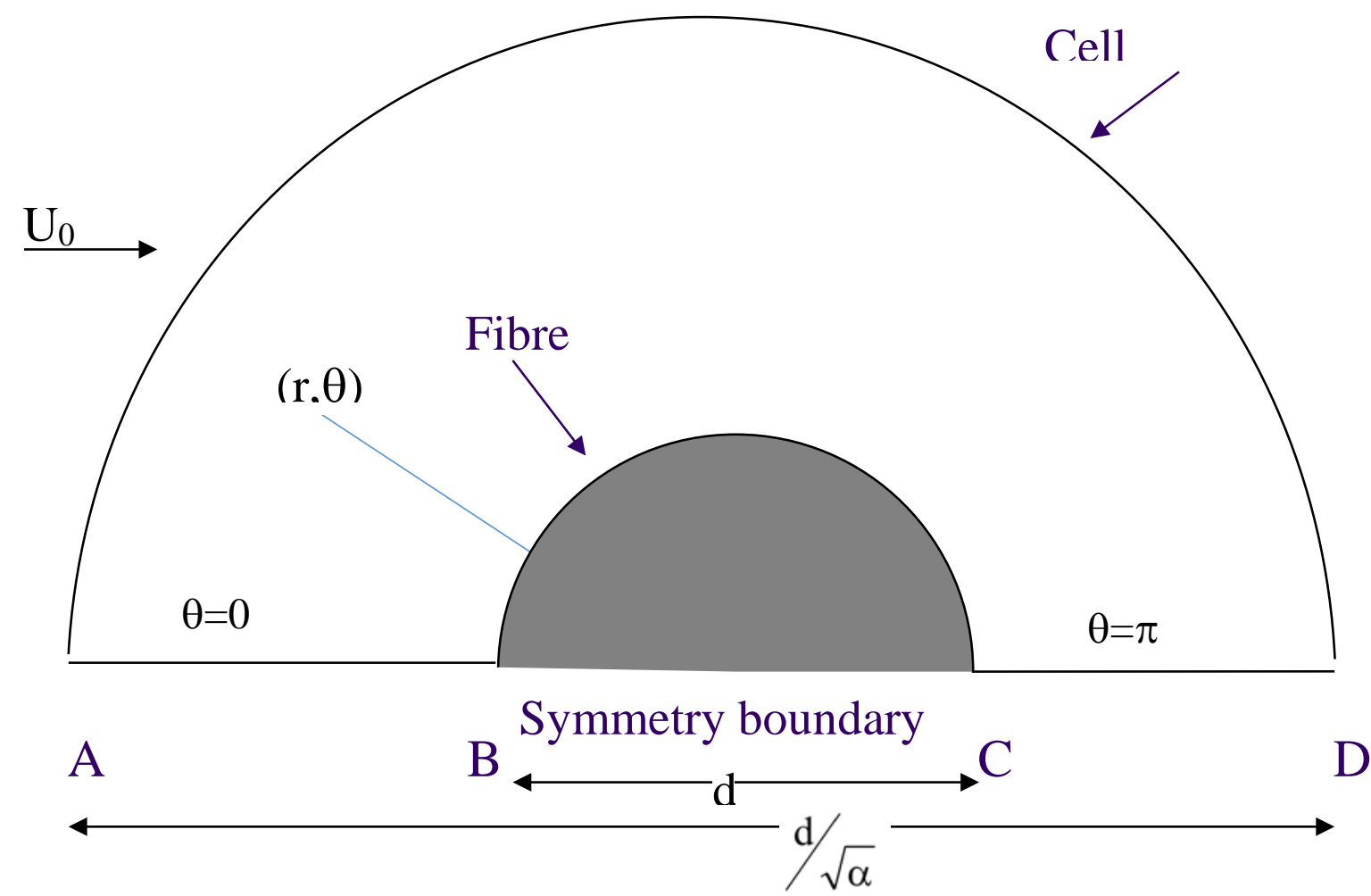

Fig. 3 Cell with fibre used for calculations of particle deposition by Dunnett and Clement (2006). 